important observation after 18 cycles is that $69 \%$ of patients had MRD negativity, which is rare with monotherapy, especially with ibrutinib".

After 12 cycles, $94 \%$ of patients aged $\geq 65$ years had a CR or CR with incomplete count recovery, and 76\% had MRD negativity in bone marrow. The estimated 1-year PFS and OS were $98 \%$ and $99 \%$, respectively.

The incidence of grade $\geq 3 \mathrm{AEs}$ was $60 \%$; treatment was discontinued in $14 \%$ of patients owing to AEs.

Several combinations of targeted

Future comparative studies will enable clinicians to determine how to best treat each patient agents have been tested in these and other trials, such as iLLUMINATE and Alliance A041202. Future comparative studies will enable clinicians to determine how to best treat each patient according to their disease features. "A head-to-head comparison of the efficacy of venetoclax and obinutuzumab versus venetoclax and ibrutinib would be reasonable," comments William Wierda, who was involved in the phase II study.

Diana Romero

ORIGINAL ARTICLE Fischer, K. et al. Venetoclax and obinutuzumab in patients with CLL and coexisting conditions. N. Engl.J. Med. $\mathbf{3 8 0}$, 2225-2236 (2019) | Jain, N. et al. Ibrutinib and venetoclax for first-line treatment of CLL. N. Engl. J.Med. 380, 2095-2103 (2019)

transplant-ineligible patients with MM. However, Rd plus bortezomib (VRd) has also been shown to have greater efficacy than Rd in this population. Moreover, daratumumab is already approved, in combination with bortezomib, melphalan and prednisone (D-VMP), for such patients. Thus, further studies are required to establish the relative benefits and drawbacks of each treatment option.

Importantly, in both the CASSIOPEIA and MAIA trials, daratumumab had only limited effects on the safety of treatment. In addition to infusion reactions known to be associated with this agent, the risks of neutropenia and lymphopenia, as well as some other toxicities, were modestly increased. David Killock

ORIGINAL ARTICLES Moreau, P. et al.

Bortezomib, thalidomide, and dexamethasone with or without daratumumab before and after autologous stem-cell transplantation for newly diagnosed multiple myeloma (CASSIOPEIA): a randomised, open-label, phase 3 study. Lancet https://doi.org/10.1016/S0140-6736(19)31240-1 (2019) | Facon, T. et al. Daratumumab plus lenalidomide and dexamethasone for untreated myeloma. N. Engl.J. Med 380, 2z104-2115 (2019)

\section{Colorectal CANCER}

\section{Microenvironment plasticity confers cetuximab resistance}

Patients with metastatic and RAS-wild-type colorectal cancer (CRC) typically receive the anti-EGFR-antibody cetuximab, either with or without chemotherapy. Acquired resistance to cetuximab is virtually inevitable and acquired mutations in RAS/RAF and EGFR have been thought to be the main drivers. Now a prospective analysis reveals

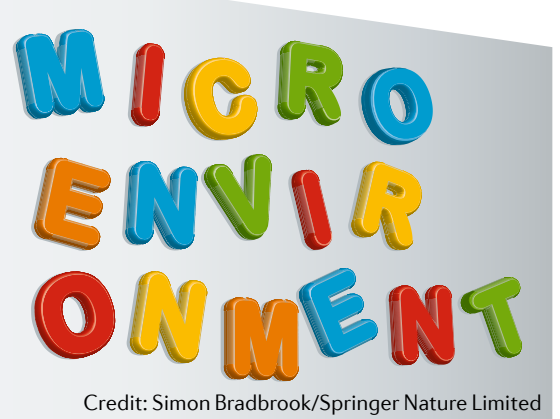
the prominent contribution of nongenetic alterations.

Lead author Marco Gerlinger explains: "published studies of acquired cetuximab resistance mainly involved patients with very good or long initial responses, which we don't often see in the clinic." Therefore: "we set up a prospective trial to investigate resistance. We were hoping that studying the molecular changes that occur when cells develop cetuximab resistance would reveal opportunities to target resistant tumours". Biopsy samples were obtained from 35 patients prior to treatment with cetuximab, with matched samples and following disease progression from 24.

Biallelic NF1 mutations and non-canonical RAS and RAF mutations were associated with primary resistance and their functional role in resistance was confirmed in vitro. At least one probable resistance mutation was detected in pretreatment biopsy samples from 16 of 20 patients with disease progression on or prior to the first follow-up CT scan ( 12 weeks after commencing therapy). Conversely, genomic alterations associated with resistance could only be detected in biopsy samples from 5 of 14 patients upon disease progression following an initial response. Deep sequencing of selected driver mutational hotspots and analysis of ctDNA revealed that genetic drivers of acquired resistance were often subclonal and that resistance remained unexplained in a large fraction of the cancer cell population.

Investigations of the transcriptomic features of tumours that acquired resistance to cetuximab without resistance mutations revealed a phenotypic switch from consensus molecular subtype 2 (CMS2) to CMS4 in 5 of 7 samples, an effect that was not observed in any of 6 patients with early disease progression. Transition to the CMS4 subtype was associated with increased release of mitogenic growth factors and a greater abundance of cancer-associated fibroblasts in biopsy samples. No underlying genetic alterations associated with phenotype switching were detected.

Analysis of immune cell infiltrates revealed increases in several immune cell types, including $\mathrm{T}_{\mathrm{H}} 1$ cells and $\mathrm{CD} 8^{+} \mathrm{T}$ cells, primarily in the centre of the tumour. No changes in neoantigen or mutational burden were observed. These changes were accompanied by increased expression of immune-checkpoint proteins, including LAG3, PD-L1, TIM3 and GITR.

Gerlinger summarizes: "Cetuximab resistance occurs not only through Darwinian acquisition of RAS-RAF pathway mutations, but also via microenvironmental plasticity, which seems to be an equally powerful driver." Notably, "the strong effect of cetuximab on the immune landscape shows that systemic therapies can change immune infiltrates quite dramatically and we hope we can leverage this to develop better immunotherapies".

Peter Sidaway 